\title{
Die Medizin von morgen in den Händen der Ärzte von heute
}

\author{
Valérie Barbiéa, Aitana Lebrand ${ }^{a}$, Maïa Berman ${ }^{b}$
}

SIB Schweizerisches Institut für Bioinformatik - ${ }^{a}$ Gruppe Klinische Bioinformatik; ${ }^{b}$ Kommunikationsabteilung

Krebs, Erb- oder Infektionskrankheiten: Schon etliche Patienten profitieren von den Fortschritten in der personalisierten Medizin. Es stellen sich aber auch neue Herausforderungen bei der Umsetzung dieser technologischen Fortschritte in die Arztpraxis. Überblick und Lösungsansätze für Ärzte in der Schweiz.

\section{Personalisierte Medizin: Versprechen und aktuelle Lage}

Hausärzte haben schon immer einen personalisierten Ansatz gewählt, weil sie den Lebenswandel und die Gesundheitsgeschichte ihrer Patienten kennen. Neue Techniken, die in jüngster Zeit zur Verfügung stehen, werden es ihnen aber auch ermöglichen, langfristig Informationen aus dem molekularen Profil ihres Patienten zu gewinnen, um Krankheiten frühzeitig zu erkennen oder eine Behandlung anzupassen.

\section{Herausforderungen und Lösungen für Ärzte}

Enorme Mengen an Daten, generiert durch neue Technologien, und die schnelle Entwicklung derselben haben schon heute das klinische Ökosystem auf den Kopf gestellt: Neue Berufe, wie zum Beispiel der des klinischen Bioinformatikers, wurden unentbehrlich in den Analyselabors, während Spitäler einen neuen Bedarf an IT-Infrastrukturen haben.

Die sich daraus entfaltende Interdisziplinarität generiert eine Nachfrage nach der Entwicklung einer gemeinsamen Sprache für die diversen Experten - Onkologen, Pathologen, Genetiker, Bioinformatiker - sowie nach einer Weiterbildung des medizinischen Fachpersonals.

Das SIB Schweizerisches Institut für Bioinformatik (siehe 1) hat sich zur Aufgabe gemacht, dieser Nachfrage gerecht zu werden, und arbeitet Hand in Hand mit den Ärzten, um ihre Bedürfnisse besser zu ver- stehen und anzugehen, zum Beispiel durch die $\mathrm{Zu}$ sammenarbeit mit Spitälern, um Prozeduren und Datenstandards zu vereinheitlichen (siehe 2); durch die Entwicklung von Datenanalysewerkzeugen zur Optimierung von Routinediagnosen; oder durch die Schaffung eines Zertifikats in Personalisierter Molekularer Onkologie mit den Universitätsspitälern von Basel und Lausanne (siehe 3).

\footnotetext{
1. Über das SIB

Das Institut ist eine gemeinnützige Organisation, die 70 Forschungs- und Dienstleistungsgruppen in der Bioinformatik in der Schweiz verbindet. Das SIB bietet Forschern und Ärzten führende Infrastruktur, Resourcen, Know-how und Ausbildung in Bioinformatik: www.sib.swiss.
}

2. Patientendaten - eine Knacknuss für Forscher

Mit Ausblick auf klinische Anwendungen befindet sich die Forschung in voller Expansion. Dabei gibt es aber auch weitere wichtige Herausforderungen zu lösen, darunter die Verwendung von Patientendaten. Der Bund hat 2017 eine nationale Initiative lanciert, welche diese Daten Forschungsprojekten zur Verfügung stellt: das Swiss Personalized Health Network (SPHN). Dem SIB wurde die Aufgabe zuteil, die Standards zum Datenaustausch zu etablieren sowie auch eine gesicherte IT-Infrastruktur für die Forschungsprojekte aufzustellen.

3. Mit einem Certificate of Advanced Studies (CAS) weitergehen Als erstes seiner Art in der Schweiz ist das CAS in Personalisierter Molekularer Onkologie offen für das gesamte Personal im Gesundheitswesen, das sich Wissen über die Methoden zur Generierung, Analyse und Interpretation des molekularen Profils von Patienten aneignen oder sein Wissen erweitern und sich mit den dazugehörigen Herausforderungen auseinandersetzen möchte. Die erste Runde beginnt im November 2018. Einschreibung und detaillierte Informationen: www.pmo.unibas.ch 